\title{
Das Allgemeine hat es schwer
}

\section{Werner Bauer}

Dr. med., Mitglied der Redaktion

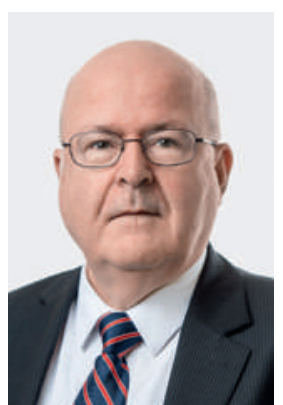

Der Titel ist auf den ersten Blick vielleicht schwer verständlich. Manche Leser denken wohl, dass das Allgemeine doch so gut wie das Spezielle seinen definierten und akzeptierten Platz in unserer Welt hat: die Allgemeinheit, das Allgemeinwohl, die allgemeinen Geschäftsbedingungen, der Allgemeinplatz - wo liegt denn das Problem?

Zumindest zwei «Allgemeinheiten» geben Stoff für Diskussionen: Wie werden die allgemeinen Aspekte in den verschiedenen medizinischen Fachgebieten künftig wahrgenommen, und wie können die allgemeinen Lernziele in der ärztlichen Bildung vermittelt werden? Niemand bestreitet, dass das Gesundheitswesen neben den Spezialärzten auch Allgemeinärzte braucht, um Erkrankungen integriert zu beurteilen, den Patienten insgesamt gerecht zu werden und nicht ausschliesslich auf ein Organ oder einen Befund zu fokussieren. Für die häufigen polymorbid Erkrankten und für komplexe Krankheitsbilder müssen genügend kompetente Generalisten zur Verfügung stehen, welche die Abklärungsund Behandlungsstrategien unter Beachtung von Prioritäten und im koordinierenden Kontakt mit den Spezialisten festlegen und umsetzen. Dies gilt nicht nur für die internistisch orientierte ärztliche Grundversorgung, sondern auch für andere Fachbereiche, wo Subspezialisierungen zweifellos notwendig sind, wo aber in vielen Fällen die Indikationsstellung für die Anwendung bestimmter Abklärungs- und Therapiemethoden von einem breiten Blick auf die zur Verfügung stehenden Möglichkeiten und auf die Gesamtsituation des Patienten begleitet sein muss. Es stellt sich auch die Frage, inwieweit für die Fachspezialisten selber heute noch eine generalistische Basisweiterbildung notwendig ist und inwieweit die Grundversorgung in verschiedensten Disziplinen ausserhalb der Zentralspitäler durch Ärzte sichergestellt werden muss, deren Rucksack mit genügender allgemeiner Kompetenz gefüllt ist.

Und jetzt zeigt sich die Berechtigung der Feststellung im Titel: Die Spezialisierung als Berufsziel gewinnt mehr und mehr an Beliebtheit, das Allgemeine hat es schwer. In der Theorie abwechslungsreich, herausfordernd, interessant und notwendig, leidet der Allgemeinbereich in der Inneren Medizin, in der Chirurgie, in der Gynäkologie und in anderen Fächern unter der Realität mühsamerer Arbeitsbedingungen, einer tieferen Einstufung in der Prestigeskala und den weniger attraktiven Saläraussichten. Um eine ehrliche Grundsatzdebatte und die sich daraus ergebenden Massnahmen kommen wir da nicht herum!

Die schwierige Umsetzung der allgemeinen Lernziele ist zwar ein zweites Thema, hat aber einen gewissen logischen Zusammenhang mit der Diagnose schwieriger Zeiten für die Generalisten. Diese Lernziele stehen zwar weltweit in den ärztlichen Weiterbildungsprogrammen und ergänzen die fachlichen Lernziele der einzelnen Gebiete. Es mag sein, dass man in den Jahrzehnten der enormen technischen und methodischen Fortschritte, die sich ja vor allem innerhalb der Spezialfächer entwickelt haben, die allgemeinen Anforderungen an eine Arztpersönlichkeit in Aus- und Weiterbildung etwas aus den Augen verloren hat. Dies hängt wohl auch damit zusammen, dass man vom Lernen am Vorbild als paternalistischer Weiterbildungssituation abgerückt ist und sich auf definierbare «skills» und «knowledge» konzentrierte. Heute finden allgemeine Kompetenzen als Grundlagen für eine umfassend gute Berufsausübung (im Englischen mit dem schwer übersetzbaren Wort professionalism umschrieben) wieder zunehmend Beachtung. Es geht um Kommunikation, Arbeit im Team, leadership, Umgang mit Fehlern, didaktische Fähigkeiten, ethische und ökonomische Grundlagen der Entscheidungsfindung, medical humanities und damit auch um den Link von Medizin und Kultur bis möglicherweise hin zur Philosophie. Eine breit angelegte Umfrage unter Absolventen der Facharztweiterbildung ermittelte kürzlich in diesen Bereichen ein Defizit. Auch hier bekommt der Titel recht: Das Allgemeine hat es schwer. Eine ganze Reihe von Organisatoren hat die Erfahrung gemacht, dass zwar die Wichtigkeit der allgemeinen Kompetenzen verbal eifrig betont wird, dass aber der Besuch entsprechender Lehrveranstaltungen enttäuschend ist. Es gilt dringend, Angebote zu entwickeln, die auch das Allgemeine attraktiv machen.

Das SIWF hat die Initiative ergriffen und bietet zusammen mit dem Royal College of Physicians of London auch im kommenden April und September am Kantonsspital Luzern höchst empfehlenswerte Workshops zu Themen wie Leadership for doctors, Supporting the underperforming trainee und A masterclass on effective teaching skills an (Information und Anmeldung: mihriye.habermacher[at]luks.ch). 